\title{
Fibroelastoma papilar valvular aórtico: causa atípica de ictus
}

\author{
Aortic valve papillary fibroelastoma: atypical cause of stroke \\ Carlos Domínguez-Massa*, Tomás Heredia-Cambra, José A. Rincón-Almanza, María J. Dalmau-Sorlí, \\ Francisco J. Valera-Martínez y Juan B. Martínez-León \\ Servicio de Cirugía Cardiovascular, Hospital Universitario y Politécnico La Fe, Valencia, España
}

\section{Introducción}

Los tumores primarios cardiacos son raros (incidencia del $0,001-0,3 \%$ ), siendo los secundarios más habituales (incidencia del 2-18\%). Los tumores cardiacos primarios se dividen en benignos, malignos y de comportamiento incierto, y entre ellos los más frecuentes son los benignos. El sarcoma es el tumor primario maligno más frecuente en los adultos, y el mixoma es el benigno más habitual1-4. El fibroelastoma papilar es el segundo tumor primario más frecuente, también benigno. Pueden ser asintomáticos o con manifestaciones heterogéneas, por obstrucción, embolia o crecimiento. No hay guías de manejo bien establecidas y en la mayoría de las ocasiones se requiere un equipo multidisciplinario ${ }^{1,2}$.

\section{Descripción del caso}

Varón de 52 años que presentó afasia motora, hemiplejia derecha y hemianopsia homónima derecha, por lo que se activó el código ictus. La angiografía por tomografía cerebral evidenció hipodensidad con pérdida de diferenciación córtico-subcortical frontal, sin colecciones hemáticas, con defecto de repleción de la arteria cerebral media izquierda distal y pérdida de colateralidad pial en el territorio cortical anterior. Fue tratado con fibrinólisis intravenosa, $\sin$ complicaciones.

Se iniciaron tratamiento anticoagulante y estudio etiológico. La ecocardiografía transtorácica objetivó una tumoración de pequeño tamaño en el velo coronariano derecho, sobre válvula aórtica trivalva, sin disfunción valvular y sin otras alteraciones. La ecocardiografía transesofágica confirmó la tumoración vibrátil en la cara aórtica del velo coronariano derecho, de $0.5 \mathrm{~cm}$, sugestiva de fibroelastoma papilar, sin flujos patológicos (Fig. 1). Se descartaron comunicación interauricular y trombos en la orejuela izquierda. Pasada la fase aguda del ictus, se realizó resonancia magnética cerebral, sin evidencia de complicación hemorrágica, con afectación del córtex motor extensa. Residualmente, presentaba hemiparesia derecha y disfasia motora. Se completó el estudio con coronariografía, que descartó lesiones, y se decidió realizar cirugía.

Se llevó a cabo la intervención con abordaje por miniesternotomía parcial superior. Se encontró la tumoración valvular aórtica de superficie blanca, fibrosa y friable, anclada por un pedículo a la cara aórtica de la porción libre del velo coronariano derecho, de $0.5 \mathrm{~cm}$ de diámetro, y se resecó (Fig. 2). Por su forma, tamaño
Correspondencia:

${ }^{*}$ Carlos Domínguez-Massa

E-mail: dominguez.massa@gmail.com
Fecha de recepción: 26-08-2020 Fecha de aceptación: 31-01-2021 DOI: 10.24875/ACM.200004041
Disponible en internet: 10-03-2021 Arch Cardiol Mex. 2021;91(4):528-530 www.archivoscardiologia.com 1405-9940 / @ 2021 Instituto Nacional de Cardiología Ignacio Chávez. Publicado por Permanyer. Este es un artículo open access bajo la licencia CC BY-NC-ND (http://creativecommons.org/licenses/by-nc-nd/4.0/). 


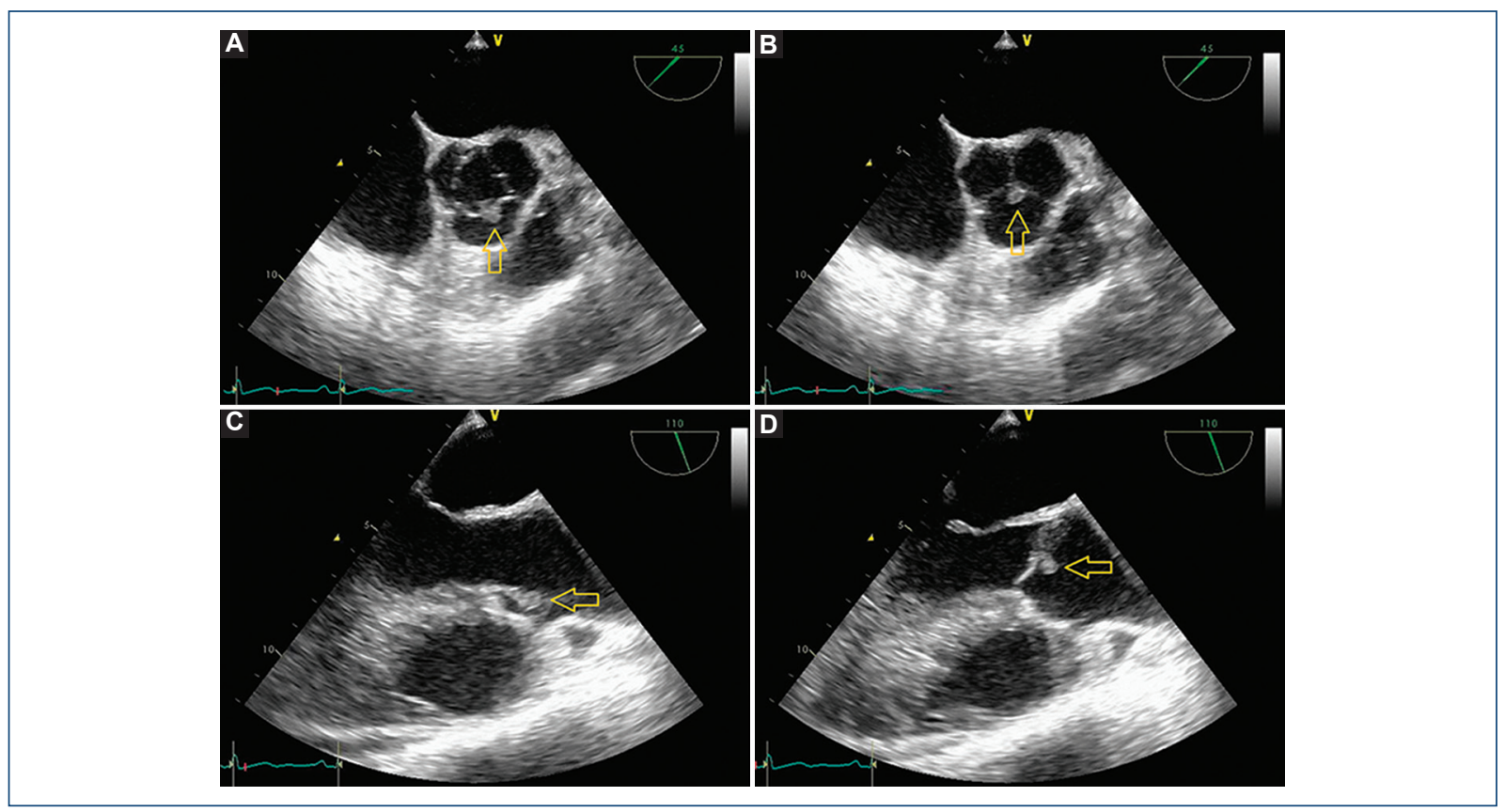

Figura 1. Ecocardiografía transesofágica preoperatoria que muestra la tumoración (flecha). A: corte transversal basal, apertura de válvula aórtica. B: válvula aórtica cerrada. C: corte medio transversal de cinco cámaras, válvula aórtica abierta. D: válvula aórtica cerrada.

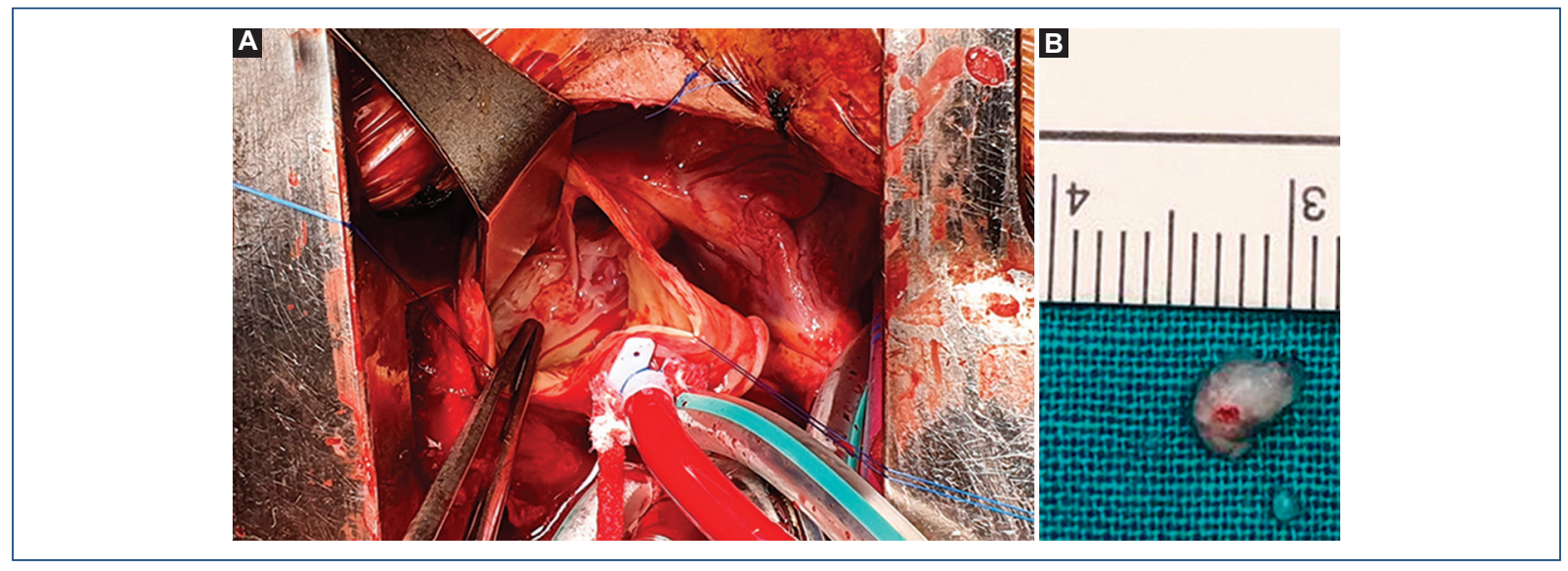

Figura 2. Fotografías quirúrgicas. A: tumoración valvular aórtica anclada al velo coronariano derecho. B: tumoración resecada.

y apariencia similar a una anémona de mar descrita en la literatura al sumergirla en solución salina fisiológica, se consideró sugestiva de fibroelastoma papilar, y se decidió conservar la válvula aórtica ${ }^{5,6}$. El paciente tuvo una excelente evolución posoperatoria y fue dado de alta al séptimo día. La ecocardiografía transtorácica de control no mostró alteraciones, sin flujos patológicos. Microscópicamente, la muestra quirúrgica presentaba una arquitectura papilar conformada por pequeñas frondas avasculares centradas por un estroma laxo escasamente celular de elementos ovales de núcleo pequeño, basófilo, con citoplasmas poco definidos, positivas para vimentina y S-100, y negativas para calretinina. Estaban revestidas por una hilera de células endoteliales CD31 positivas, sin signos de malignidad, y se confirmó el diagnóstico de fibroelastoma papilar. 


\section{Discusión}

Los tumores primarios cardiacos son infrecuentes, y de ellos, el $70-75 \%$ son benignos ${ }^{1,2,4}$. El fibroelastoma papilar es el segundo tumor primario más frecuente (10\% de todos los tumores cardiacos). A pesar de que el mixoma es el más habitual según la literatura, en la actualidad el fibroelastoma papilar sobrepasa al mixoma como lesión cardiaca más comúnmente resecada. Tiene predominio en el sexo femenino, con una edad de presentación de alrededor de 65 años $^{5}$. Puede originarse en cualquier zona del endocardio, pero lo más común es la afectación cardiaca izquierda. Tiene preferencia de implantación sobre las válvulas cardiacas, siendo la aórtica la más afectada (sobre todo el seno no coronariano), seguida por la mitral, el endocardio ventricular izquierdo y la válvula tricúspide ${ }^{1,4-6}$. Habitualmente son tumores aislados, pero pueden ser múltiples².

Existen pocos estudios de seguimiento a largo plazo. Se ha reportado un crecimiento tumoral de $0.47 \mathrm{~mm}$ por año cuando se localiza en la válvula aórtica, y menor en la válvula mitral ${ }^{5}$. Macroscópicamente parecen una fronda con múltiples proyecciones fibrosas con apariencia de anémona de mar al sumergirlo en sue$\mathrm{ro}^{6,7}$. Microscópicamente tienen un núcleo avascular fibroelástico delimitado por endocardio ${ }^{6}$. Se han descrito como hamartomas o trombos organizados por presentar fibrina, ácido hialurónico y fibras elásticas entre las frondas, asociándose a anomalías congénitas. Otra hipótesis etiológica es la traumática o iatrogénica por lesión mecánica o daño endotelial posradiación ${ }^{4}$.

La mayoría cursan asintomáticos y son un hallazgo casual en una prueba de imagen o una cirugía por otra causa. La clínica es consecuencia de su embolia, con frecuencia cerebrovascular con accidentes isquémicos. Pueden dar embolias periféricas y obstrucción de los ostia coronarios, y debutar como infarto de miocardio, sobre todo en la válvula aórtica ${ }^{4,6,8}$. Debe realizarse el diagnóstico diferencial con coágulos, calcio, vegetaciones $u$ otros cuerpos extraños ${ }^{2}$.

Su manejo no está bien establecido y depende de su presentación. Se recomienda la resección en los pacientes sintomáticos a pesar del tamaño y en pacientes sometidos a cirugía cardiaca por otro motivo, pero no hay consenso en cuanto a los pacientes asintomáticos ${ }^{6,9}$. Debe considerarse la cirugía cuando la lesión es izquierda, por alto riesgo embolígeno, y se valorará de forma individual en la afectación derecha. Dado el bajo riesgo quirúrgico, suele realizarse la resección de las lesiones derechas mayores de $1 \mathrm{~cm}^{2,9}$.
Podrían tratarse de manera conservadora con anticoagulación las lesiones menores de $1 \mathrm{~cm}$ no móviles, aunque no está validada la eficacia del tratamiento anticoagulante ${ }^{6-9}$.

No suelen producir afectación funcional valvular tras su resección ${ }^{2}$. Tampoco suelen recidivar, con limitados casos descritos en la bibliografía (7,5\% a 2 años), por lo que en la mayoría de los casos se considera conservar la válvula afecta si se puede resecar la tumoración con garantías ${ }^{5,8,9}$.

\section{Conclusión}

Se recomienda el manejo de estas lesiones en centros especializados, con un equipo multidisciplinario, individualizando la toma de decisiones según la sospecha diagnóstica inicial y la afectación ${ }^{2}$. Se debe garantizar un seguimiento estrecho para poder detectar recidivas ${ }^{5}$.

\section{Conflicto de intereses}

Ninguno.

\section{Responsabilidades éticas}

Protección de personas y animales. Los autores declaran que para esta investigación no se han realizado experimentos en seres humanos ni en animales.

Confidencialidad de los datos. Los autores declaran que en este artículo no aparecen datos de pacientes.

Derecho a la privacidad y consentimiento informado. Los autores declaran que en este artículo no aparecen datos de pacientes.

\section{Bibliografía}

1. Burke A, Tavora F. The 2015 WHO classification of tumors of the heart and pericardium. J Thorac Oncol. 2016;11:441-52.

2. Yanagawa B, Mazine A, Chan EY, Barker CM, Gritti M, Reul RM, et al. Surgery for tumors of the heart. Semin Thoracic Surg. 2018;30:385-97.

3. Domínguez-Massa C, Bel-Mínguez AM, Montero-Argudo JA. Presentación atípica de mixoma auricular gigante. Cir Cardiov. 2018;25:45-7.

4. Saleh WKA, Al Jabbari O, Ramlawi B, Reardon MJ. Cardiac papillary fibroelastoma: single-institution experience with 14 surgical patients. Tex Heart Inst J. 2016;43:148-51.

5. Kurmann R, El-Am E, Bois M, Scott C, Lee A, Sorour A, et al. Clinical and echocardiographic characteristics in patients with pathology proven cardiac papillary fibroelastomas. Eur Heart J. 2019;40:278.

6. Rodrigues JD, Ferreira J, Almeida J, Campelo M, Maciel MJ, Pinho P. Cardiac papillary fibroelastoma: report of a surgical series. Rev Port Cardiol. 2019;37:981-6.

7. Taha ME, Kumaresan J. Aortic valve papillary fibroelastoma: a sea anemona in the heart, case report. Cardiol Res. 2019;10:378-81.

8. Logan N, Islam MS, Chughtai JZ, Murphy NF. An atypical causa of myocardial infarction: case report of an obstructing papillary fibroelastoma of the aortic valve. Eur Heart J Case Rep. 2019;3:ytz058.

9. Ikegami H, Andei AC, Li Z, McCarthy PM, Malaisrie SC. Papillary fibroelastoma of the aortic valve: analysis of 21 cases, including a presentation with cardiac arrest. Tex Heart Inst J. 2015;42:131-5. 